\title{
Mesons in Nuclei in the Light-Front Mean Field Approximation and Deep-Inelastic Scattering
}

\author{
M. Burkardt \\ Department of Physics \\ New Mexico State University \\ Las Cruces, NM 88003-0001 \\ U.S.A. \\ G. A. Miller \\ Department of Physics, Box 351560 \\ University of Washington \\ Seattle, WA 98195-1560 \\ U.S.A.
}

(November 11, 2018)

A light-front treatment for the scalar and vector meson momentum distribution functions is developed using a model in which the nucleus is treated a static source of radius $R$. The limit $R \rightarrow \infty$ corresponds to infinite nuclear matter. In this limit, the scalar mesons are shown to carry a vanishing momentum fraction of the nuclear plus momentum $k^{+}$, but the vector mesons carry a significant fraction, all occuring at the experimentally inaccessible value of $k^{+}=0$. This confirms earlier work. A study of the $R$ dependence for sizes of real nuclei shows that a fraction of the scalar and vector mesons could be observable.

\section{INTRODUCTION}

It has been known that there is a significant difference between the parton distributions of free nucleons and nucleons in a nucleus, since the famous EMC experiment [1]. The EMC-effect can be explained if the momentum distribution of valence quarks is slightly shifted towards smaller values of $x$ for nucleons in a nucleus. One way to achieve this result is by having mesons carry a larger fraction of the momentum in the nucleus [2]. While such a model explains the shift in the valence distribution, one obtains at the same time a meson (i.e. anti-quark) distribution in the nucleus, which is strongly enhanced compared to free nucleons and which should be observable in Drell-Yan experiments [3]. However, no such enhancement has been observed experimentally [ $⿴$. The significance of this absence has been emphasized in Ref. [5].

The so-called EMC effect is a subtle one. For example, its size is typically about $10 \%$ which is of the same order as relativistic effects. Motivated by the need to include a relativistic treatment of the motion of the nucleons, which is also consistent with the information derived using conventional nuclear dynamics, one of us attempted to construct a light front treatment of nuclear physics
[6]. The light front is relevant because structure functions depend on the Bjorken variable which is a ratio of the plus-momentum $k^{0}+k^{3}$ of a quark to that of the target. The calculations of Ref. [6], using a Lagrangian in which Dirac nucleons are coupled to massive scalar and vector mesons [7], treated the example of infinite nuclear matter within the mean field approximation. In this case, the meson fields are constants in both space and time. This means that the momentum distribution has support only at $k^{+}=0$. Such a distribution would not be accessible experimentally, so that the suppression of the plus-momentum of valence quarks would not imply the existence of a corresponding testable enhancement of anti-quarks. However, it is necessary to ask if the result is only a artifact of the infinite nuclear size and of the mean field approximation.

In this note, we investigate the dependence on nuclear size by explicitly constructing the scalar and vector meson states for a model in which the nucleus is represented as a static source of radius $R$ and mass $M_{A}$. Since $M_{A}$ is very large for large $A$, the nucleus acts as an extended static source. It should be emphasized that we do not assume that individual nucleons are infinitely heavy to obtain this result. In the mean field approximation, the nucleus acts as a static source if it is infinitely heavy. Then a coherent state is the ground state of the Hamiltonian. The organization of the paper is as follows. The model for the treatment of scalar mesons is defined using the rest-frame (equal time formulation) in Sect. II. The light front LF treatment of that system is made in Sect. III, in which the plus-momentum distribution function is computed. We study vector mesons in the LF treatment in Sect. IV, and the distribution functions are obtained. The separate treatment of scalar and vector meson effects is made to simplify the discussion. Sect. V contains a summary of the results and their possible implications. The derivation of a term in the light front Hamiltonian that includes the recoil momentum of the 
heavy source is made in an Appendix.

\section{SCALAR MESON DISTRIBUTION IN THE REST-FRAME FORMULATION}

Before we proceed to the LF formulation, let us first analyse the scalar meson distribution in the mean field approximation in a rest frame. This simple calculation is of help in understanding the LF calculation.

Our model here is that of scalar mesons, coupled to a large static nucleus represented by a scalar source $J(\vec{r})$. Such a system is described by the Lagrangian density

$$
\mathcal{L}=\frac{1}{2} \partial_{\mu} \phi \partial^{\mu} \phi-\frac{m_{S}^{2}}{2} \phi^{2}+J \phi .
$$

Canonical quantization proceeds by using

$$
\phi(\vec{r})=\int \frac{d^{3} k}{(2 \pi)^{3 / 2} \sqrt{2 \omega_{\vec{k}}}}\left[a_{\vec{k}} e^{i \vec{k} \cdot \vec{r}}+a_{\vec{k}}^{\dagger} e^{-i \vec{k} \cdot \vec{r}}\right],
$$

where $\omega_{\vec{k}}=\sqrt{m_{S}^{2}+\vec{k}^{2}}$. Then the Hamiltonian reads

$$
H=\sum_{\vec{k}}\left[\omega_{\vec{k}} a_{\vec{k}}^{\dagger} a_{\vec{k}}-\frac{1}{\sqrt{2 \omega_{\vec{k}}}}\left(\tilde{J}^{*}(\vec{k}) a_{\vec{k}}^{\dagger}+\tilde{J}(\vec{k}) a_{\vec{k}}\right)\right]
$$

where

$$
\tilde{J}(\vec{k})=\tilde{J}^{*}(-\vec{k})=(2 \pi)^{-3 / 2} \int d^{3} r e^{-i \vec{k} \cdot \vec{r}} J(\vec{r}) .
$$

As a specific model, let us consider a spherical nucleus of radius $R$ with constant density, i.e.

$$
J(\vec{r})=J_{0} \Theta(R-|\vec{r}|) .
$$

The quantity $J_{0}$ can be thought of as arising from the product of a scalar meson coupling constant $g_{S}$ and an appropriate scalar density $\rho_{S}$ so that

$$
J_{0}=g_{S} \rho_{S} .
$$

The ground state of $H$ (2.3) can be obtained by means of a coherent state ansatz, with the result

$$
\left|\psi_{0}\right\rangle \propto\left[\prod_{\vec{k}} \exp \left(\frac{\tilde{J}^{*}(\vec{k}) a_{\vec{k}}^{\dagger}}{\sqrt{2 \omega_{\vec{k}}^{3}}}\right)\right]|0\rangle
$$

This state is an eigenfunction of $H$ with eigenvalue (binding energy)

$$
E_{0}=-\frac{1}{2} \int d^{3} k \frac{|\tilde{J}(\vec{k})|^{2}}{\omega_{\vec{k}}^{2}}
$$

This can be re-expressed in terms of an integral over coordinate space:

$$
E_{0}=-\frac{1}{8 \pi} \int d^{3} r d^{3} r^{\prime} J(\vec{r}) \frac{e^{-m_{S}\left|\vec{r}-\vec{r}^{\prime}\right|}}{\left|\vec{r}-\vec{r}^{\prime}\right|} J\left(\vec{r}^{\prime}\right) .
$$

A brief examination of Eq. (2.9) shows that for values of $m_{S} R \gg 1, E_{0}$ is approximately proportional to $J_{0}^{2} \frac{4 \pi}{3} R^{3}$ times the volume integral of the Yukawa function, which means that, as expected, the binding energy is proportional to the number of nucleons. This result would also be obtained if the theta function of Eq. (2.5) were replaced by a smoother function that incorporated a nuclear surface thickness. One sees also the usual result that the nucleus would not be stable (the energy per nucleon would have no minimum as a function of $R$ ) using scalar mesons alone.

One may proceed to evaluate $E_{0}$ by using straightforward contour integration techniques with the result

$$
\begin{aligned}
E_{0}= & -\frac{1}{2}\left(g_{S} \rho_{S}\right)^{2}\left[\frac{4 \pi}{3} \frac{R^{3}}{m_{S}^{2}}-2 \pi \frac{R^{2}}{m_{S}^{3}}\left(1-\frac{1}{m_{S}^{2} R^{2}}\right)\right] \\
& -\frac{1}{2}\left(g_{S} \rho_{S}\right)^{2}\left[-2 \pi \frac{R^{2}}{m_{S}^{3}} e^{-2 m_{S} R}\left(1+\frac{1}{m_{S} R}\right)^{2}\right] \\
& \stackrel{R \rightarrow \infty}{\longrightarrow}-\frac{1}{2}\left(g_{S} \rho_{S}\right)^{2} \frac{4 \pi}{3} \frac{R^{3}}{m_{S}^{2}} .
\end{aligned}
$$

The momentum distribution of scalar mesons is obtained by taking the necessary expectation value:

$$
\rho(\vec{k}) \equiv\left\langle\psi_{0}\left|a_{\vec{k}}^{\dagger} a_{\vec{k}}\right| \psi_{0}\right\rangle=\frac{|\tilde{J}(\vec{k})|^{2}}{2 \omega_{\vec{k}}^{3}} .
$$

Evaluation of Eq. (2.5) using Eq.(2.4) gives

$$
\begin{aligned}
\tilde{J}(\vec{k}) & =\sqrt{\frac{2}{\pi}} J_{0} \frac{\sin (k R)-k R \cos (k R)}{k^{3}} \\
& =\sqrt{\frac{2}{\pi}} J_{0} R^{3} \frac{j_{1}(k R)}{k R}
\end{aligned}
$$

where $k \equiv|\vec{k}|$, and $j_{1}(x)$ is the spherical Bessel function of order one: $j_{1}(x)=\sin (x) / x^{2}-\cos (x) / x$. Thus one finds

$$
\rho(\vec{k})=\frac{J_{0}^{2}}{\pi} R^{6}\left(\frac{j_{1}(k R)}{k R}\right)^{2} \frac{1}{\omega_{\vec{k}}^{3}}
$$

which is sharply peaked near $k=0$ for large nuclei, as $j_{1}(x)$ has its first zero at $x=k R=4.5$. One may compute the expectation value of the pion field in the state $\left|\psi_{0}\right\rangle$ to find

$$
\begin{aligned}
& \left\langle\psi_{0}|\phi(\vec{r})| \psi_{0}\right\rangle \\
& \quad=\frac{g_{S} \rho_{S}}{2 m_{S}^{2}}\left[2-\frac{\left(1+m_{S} R\right)}{m_{S} r} e^{-m_{S} R}\left(e^{m_{S} r}-e^{-m_{S} r}\right)\right],
\end{aligned}
$$

for $r<R$. This function is a constant, $\frac{g_{S} \rho_{S}}{2 m_{S}^{2}}$ in the limit of infinite nuclear radius in which the conditions $m_{S} R \gg 1$ and $r \ll R$ hold. 


\section{MESON DISTRIBUTION IN THE LF FORMULATION-SCALAR MESONS}

The essential observation for the LF formulation of systems coupled to static sources is that static sources in a rest-frame correspond to uniformly (constant $v^{+}=$ $\left.\left(v^{0}+v^{3}\right) / \sqrt{2}\right)$ moving sources in a LF framework [8].

The light front approach implies the following: The canonical commutation relations

$$
\left[\phi\left(x^{+}, x^{-}, \vec{x}_{\perp}\right), \partial_{-} \phi\left(x^{+}, y^{-}, \vec{y}_{\perp}\right)\right]=\frac{i}{2} \delta\left(x^{-}-y^{-}\right) \delta\left(\vec{x}_{\perp}-\vec{y}_{\perp}\right),
$$

where $x^{ \pm}=\left(x^{0} \pm x^{3}\right) / \sqrt{2}$, are satisfied by choosing

$\phi\left(x^{-}, \vec{x}_{\perp}\right)=\int_{0}^{\infty} \frac{d k^{+}}{\sqrt{4 \pi k^{+}}} \int \frac{d^{2} k_{\perp}}{2 \pi}\left[a_{k^{+} \vec{k}_{\perp}}^{\dagger} e^{i \boldsymbol{k} \cdot \boldsymbol{x}}+a_{k^{+} \vec{k}_{\perp}} e^{-i \boldsymbol{k} \cdot \boldsymbol{x}}\right]$

where $\boldsymbol{k} \cdot \boldsymbol{x}=k^{+} x^{-}-\vec{k}_{\perp} \cdot \vec{x}_{\perp}$ as we are taking $x^{+}=$ 0 . The operators $a_{k+\vec{k}_{\perp}}$ and $a_{k^{+} \vec{k}_{\perp}}^{\dagger}$ obey standard boson commutation relations consistent with Eq.(3.1).

A static charge distribution in a rest-frame $J_{R F}\left(\vec{x}_{\perp}, x^{3}\right)$ corresponds to a uniformly moving charge distribution on the LF, where at $x^{+}=0$

$$
J_{L F}\left(\vec{x}_{\perp}, x^{-}\right)=J_{R F}\left(\vec{x}_{\perp}, x^{-} v^{+}\right)=J\left(\vec{x}_{\perp}, x^{3}\right) .
$$

If the source is at rest in the rest-frame, then $v^{+}=$ $1 / \sqrt{2}$ in Eq. (3.3). This relation between static external sources in a rest-frame and moving sources on the LF is exact. In order to see how it arises, consider a time $\left(x^{0}\right)$ independent scalar field in LF variables $\left[x^{ \pm}=\left(x^{0} \pm x^{3}\right) / \sqrt{2}\right]$

$$
J_{L F}\left(\vec{x}_{\perp}, x^{-}, x^{+}\right)=J_{R F}\left(\vec{x}_{\perp}, x^{3}=\frac{x^{+}-x^{-}}{\sqrt{2}}, x^{0}=\frac{x^{+}+x^{-}}{\sqrt{2}}\right)
$$

If the field is $x^{0}$-independent, then one finds for $x^{+}=0$ : $J_{L F}\left(\vec{x}_{\perp}, x^{-}\right)=J_{R F}\left(\vec{x}_{\perp},-x^{-} / \sqrt{2}\right)$, which is Eq. (3.3) for this parity-even spatial distribution and for the special case $v^{+}=1 / \sqrt{2}$.

Using the fixed charge formalism from Refs. [8,9] one thus finds for the LF Hamiltonian of scalar fields coupled to this external source

$$
\begin{aligned}
P^{-}= & \sum_{k^{+}, \vec{k}_{\perp}}\left[\left(\frac{m_{S}^{2}+\vec{k}_{\perp}^{2}}{2 k^{+}}+\frac{k^{+}}{2 v^{+2}}\right) a_{k^{+} \vec{k}_{\perp}}^{\dagger} a_{k^{+} \vec{k}_{\perp}}\right. \\
& \left.+\frac{1}{v^{+} \sqrt{2 k^{+}}}\left(\tilde{J}_{R F}\left(\vec{k}_{\perp}, k^{+} / v^{+}\right) a_{k^{+} \vec{k}_{\perp}}^{\dagger}+\text { h.c. }\right)\right] .
\end{aligned}
$$

The term proportional to $\frac{k^{+}}{2 v^{+2}}$ arises from the plus momentum of the heavy source and its inclusion is necessary to maintain the rotational invariance of the light-front approach; its derivation is presented in the Appendix. 8

The rest-frame binding energy can be obtained using

$$
E_{R F} \equiv P^{-} v^{+}
$$

Like the rest frame Hamiltonian $H$ [Eq. (2.3)], the LF Hamiltonian $P^{-}$[Eq. (3.5)] is Gaussian in the scalar fields and the ground state is a coherent state

$\left|\psi_{0}\right\rangle_{L F} \propto\left[\prod_{k^{+}, \vec{k}_{\perp}} \exp \left(\frac{\tilde{J}_{R F}^{*}\left(\vec{k}_{\perp}, k^{+} / v^{+}\right) a_{k^{+} \vec{k}_{\perp}}^{\dagger}}{v^{+} \sqrt{2 k^{+}}\left(\frac{m^{2}+\vec{k}_{\perp}^{2}}{2 k^{+}}+\frac{k^{+}}{2 v^{+2}}\right)}\right)\right]|0\rangle$

with energy eigenvalue

$P_{0}^{-}=-\frac{1}{v+^{2}} \int_{0}^{\infty} \frac{d k^{+}}{2 k^{+}} \int d^{2} k_{\perp} \frac{\left|\tilde{J}_{R F}\left(\vec{k}_{\perp}, k^{+} / v^{+}\right)\right|^{2}}{\frac{m_{S}^{2}+\vec{k}_{\perp}^{2}}{2 k^{+}}+\frac{k^{+}}{2 v^{+2}}}$.

Note that the rest frame energy calculated from the LF result Eq. (3.8), i.e. $E_{R F}=v^{+} P_{0}^{-}$, and the binding energy calculated directly in normal coordinates [Eq. (2.8)] can be shown to be identical, as is necessary. To see this identity, make the change of integration variables: $\frac{k^{+}}{v^{+}} \rightarrow k^{3}$.

The LF-momentum distribution for the scalar mesons

$$
\rho_{S}\left(\vec{k}_{\perp}, k^{+}\right)=\left\langle\psi_{0}\left|a_{k^{+} \vec{k}_{\perp}}^{\dagger} a_{k^{+} \vec{k}_{\perp}}\right| \psi_{0}\right\rangle
$$

can be calculated using Eq. (3.7), with the result

$$
\rho_{S}\left(\vec{k}_{\perp}, k^{+}\right)=\frac{2 k^{+}\left|\tilde{J}_{R F}\left(\vec{k}_{\perp}, k^{+} / v^{+}\right)\right|^{2}}{v^{+^{2}}\left[m_{S}^{2}+\vec{k}_{\perp}^{2}+\left(\frac{k^{+}}{v^{+}}\right)^{2}\right]^{2}} .
$$

Since $\tilde{J}_{R F}\left(\vec{k}_{\perp}, k^{+} / v^{+}\right)$is strongly peaked for $k \sim 1 / R$, the momentum distribution is also trivially peaked near small momenta (for $R \gg 1 / m_{S}$ ). Physically, this is obvious since the mean field approximation gives rise to meson fields which are nearly constant throughout the nucleus. In Fourier space, a nearly constant meson field corresponds to a momentum distribution which is peaked near zero 6 .

Note that the form of $\rho_{S}\left(\vec{k}_{\perp}, k^{+}\right)$of Eq. (3.10) is different than the rest-frame momentum distribution $\rho(\vec{k})$ of Eq. 2.13). The light frame momentum distribution function and the plus-momentum distribution obtained by integrating this quantity over all values of $\vec{k}_{\perp}$ can only be obtained using the light front formulation.

A quantity of great interest is the total LF momentum carried by the meson field. A naive estimate of this quantity would yield momenta that are of the order of the (rest-frame) binding energy times the velocity, i.e. 


$$
\left\langle k^{+}\right\rangle_{\text {naive }} \approx v^{+}\left|E_{R F}\right| .
$$

We will now show that this naive estimate is wrong in the mean field approximation.

Using Eq. (3.10), one finds

$$
\begin{aligned}
\left\langle k^{+}\right\rangle & \equiv \int d^{2} k_{\perp} \int_{0}^{\infty} d k^{+} \rho\left(\vec{k}_{\perp}, k^{+}\right) k^{+} \\
& =\int d^{2} k_{\perp} \int_{0}^{\infty} d k^{+} \frac{2 k^{+2}\left|\tilde{J}_{R F}\left(\vec{k}_{\perp}, k^{+} / v^{+}\right)\right|^{2}}{v^{+^{2}}\left[m_{S}^{2}+\vec{k}_{\perp}^{2}+\left(\frac{k^{+}}{v^{+}}\right)^{2}\right]^{2}} \\
& =v^{+} \int d^{3} k \frac{k_{z}^{2}\left|\tilde{J}_{R F}\left(\vec{k}_{\perp}, k_{z}\right)\right|^{2}}{\left[m_{S}^{2}+\vec{k}^{2}\right]^{2}} \\
& =\frac{v^{+}}{3} \int d^{3} k \frac{\vec{k}^{2}\left|\tilde{J}_{R F}(\vec{k})\right|^{2}}{\left[m_{S}^{2}+\vec{k}^{2}\right]^{2}}
\end{aligned}
$$

where we used rotational invariance of the source in the rest-frame. It is interesting to express the quantity $\left\langle k^{+}\right\rangle$in terms of a coordinate space integral analogous to Eq. (2.9) for the energy. We find:

$$
\left\langle k^{+}\right\rangle=\int d^{3} r d^{3} r^{\prime} J(\vec{r})\left[\left(1-\frac{m_{S}}{2}\left|\vec{r}-\vec{r}^{\prime}\right|\right) \frac{e^{-m_{S}\left|\vec{r}-\vec{r}^{\prime}\right|}}{12 \pi\left|\vec{r}-\vec{r}^{\prime}\right|}\right] J\left(\vec{r}^{\prime}\right) .
$$

The quantity in brackets has a volume integral of 0 (with the integration variable as $\left.\left|\vec{r}-\vec{r}^{\prime}\right|\right)$. Thus the integral receives non-vanishing contributions only from regions near the nuclear surface, as is expected from the notion that the scalar meson field would be constant for a nucleus of infinite size and so receives nonzero Fourier components only from the regions near the surface of the nucleus. This means that $\left\langle k^{+}\right\rangle \propto R^{2}$ which gives a far smaller magnitude than the $R^{3}$ behavior of the binding energy. In particular, $\frac{\left\langle k^{+}\right\rangle}{v^{+} E_{0}} \sim \frac{1}{R}$. This is in accord with Ref. [6] which shows that $\left\langle k^{+}\right\rangle$vanishes for the case of infinite nuclear matter.

We now make a more specific comparison of $\left\langle k^{+}\right\rangle$of Eq. (3.12) with $v^{+}\left|E_{R F}\right|$ of Eqs. (2.8) and (3.8) using the specific model for the source $J(\vec{r})$, Eq. (2.5). A direct evaluation of the integral yields

$$
\begin{aligned}
\left\langle k^{+}\right\rangle= & \frac{v^{+}}{3}\left(g_{S} \rho_{S}\right)^{2} \pi \frac{R^{4}}{m_{S}}\left[\frac{1}{m_{S}^{2} R^{2}}-\frac{3}{m_{S}^{4} R^{4}}\right. \\
& \left.+\frac{e^{-2 m_{S} R}}{m_{S} R}\left(1+\frac{1}{m_{S} R}\right)\left(1+\frac{3}{2 m_{S} R}+\frac{3}{2 m_{S}^{2} R^{2}}\right)\right] \\
& \stackrel{R \rightarrow \infty}{\longrightarrow} \frac{v^{+}}{3}\left(g_{S} \rho_{S}\right)^{2} \pi \frac{R^{2}}{m_{S}^{3}},
\end{aligned}
$$

so that

$$
\frac{\left\langle k^{+}\right\rangle}{v^{+}\left|E_{0}\right|}=\frac{1}{2 m_{S} R} \approx \frac{1}{20}
$$

in the limit of infinite $R$, and where the last estimate arises from taking $m_{S}=550 \mathrm{MeV}$. Thus the naive estimate fails. For large values of $m R$ we see that the energy grows like the volume of the system, while the plus momentum grows as the area.

The slow $\frac{1}{2 m_{S} R}$ approach to zero makes it interesting to study the plus-momentum distribution of scalar mesons, which is the integral of $\rho_{S}\left(k_{\perp}, k^{+}\right)$over all $\vec{k}_{\perp}$ divided by the nucleon number $4 \pi \frac{R^{3}}{3} \rho_{B}$ with $\rho_{B}$ the baryon density.

Before considering this quantity it is worthwhile to follow the usual procedure of defining a dimensionless momentum distribution function. This is done by using the $x$ variable

$$
x=\frac{k^{+}}{M_{N} v^{+}},
$$

which has the physical interpretation of the LFmomentum distribution of mesons per nucleon, and taking the nucleons plus-momentum to be $M_{N} v^{+}$, which occurs for a free nucleon at rest. More precisely, one should use $M_{N}-15.75 \mathrm{MeV}$ instead of $M_{N}$ to define the variable $x$ The latter number is the average binding energy; practically speaking this small shift does not matter and is ignored here.

The variable $x$ is the quantity of relevance for deep inelastic scattering from nuclear targets. We then have after including the Jacobian of the transformation between the variables $k^{+}$and $x$ :

$$
f_{S}(x)=\frac{3}{4 \pi R^{3} \rho_{B}} M_{N} v^{+} \int d^{2} k_{\perp} \rho_{S}\left(k_{\perp}, k^{+}\right),
$$

which can be evaluated as (Fig. 1)

$$
f_{S}(x)=M_{N}^{2} \frac{\left(g_{S} \rho_{S}\right)^{2}}{\rho_{B}} R^{5} \frac{6}{\pi} x \int_{x M_{N} R}^{\infty} \frac{d y}{\left(y^{2}+m_{S}^{2} R^{2}\right)^{2}} \frac{j_{1}^{2}(y)}{y} .
$$

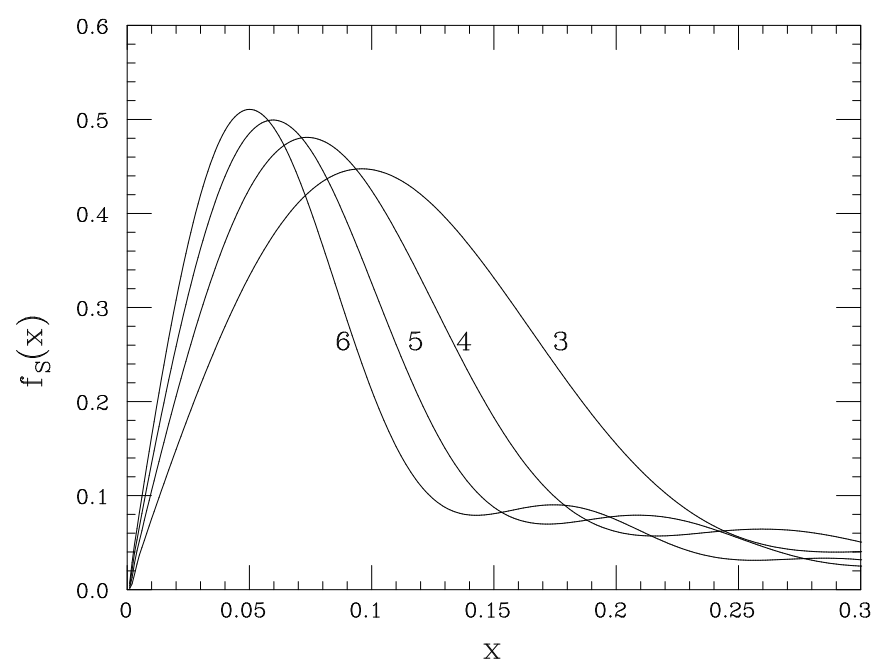

FIG. 1 . The quantity $f_{S}(x)$ vs. $x$ for nuclei of radii $3,4,5,6$ $\mathrm{fm}$ (the numbers on the curves refer to the nuclear radius ). 
It is interesting to compare the result 3.18) with previous meson distributions obtained using the equal time formulation of the nuclear wave function. These distributions exist only for pions 10,11, but the procedure outlined in Eqs.(22)-(24) of Ref. [11] can be applied to scalar mesons. Using the interaction of Eq. (2.5) and the wave function of Eq. 2.7) in those earlier formulae does indeed lead to the distribution function of Eq. (3.18). This is a consequence of the feature that the interaction of Eq. (2.5) does not excite the nucleus. Thus, in this mean field example, the equal time ground state wave function is sufficient to compute the distribution function. However, the meson distribution functions are light-like correlation functions which are equal $x^{-}$"time" correlation functions, see e.g. the reviews [9,2]. Thus it is a specific and general feature of the light front wave approach that knowing only the ground state wave function is sufficient for computing the distribution functions.

Armed with the distribution function $f_{S}(x)$ we may compute the momentum fraction carried by the nuclear scalar mesons in our mean field approximation

$$
\left\langle x_{S}\right\rangle \equiv \int_{0}^{\infty} x f_{S}(x) d x
$$

is not given by the ratio of the binding energy per nucleon to the nucleon mass $M_{N}$, but by

$$
\left\langle x_{S}\right\rangle=\frac{\left(g_{S} \rho_{S}\right)^{2}}{\rho_{B} m_{S}^{3}} \frac{3}{4 R M_{N}},
$$

in the limit of $m_{S} R \gg 1$. This quantity vanishes for nuclear matter.

It is worthwhile to study how the limit of nuclear matter is approached. The numerical results to be presented are obtained using the parameters 12]

$$
\frac{g_{S}^{2} M_{N}^{2}}{m_{S}^{2}}=267.1, \quad \rho_{S}=.179 \mathrm{fm}^{-3} .
$$

The quantity $f_{S}(x)$ is displayed in Fig.(1). One sees that $f_{S}(x)$ is fairly small for all $x$ and for all reasonable ranges of $R$. The relevant size here is 2.5 which is the value of the nucleon distribution function at its peak [6] and which also is typical of peak values of the vector meson distribution discussed below. Note that the integral of $f_{S}(x)$ does not vanish. The quantity $x f_{S}(x)$ peaks at smaller and smaller values of $x$ for larger and larger values of $R$.

A final point concerns the expectation value of the scalar field. Defining the expectation value of the scalar field of the expansion Eq. 3.2 in the state $\left|\psi_{0}\right\rangle_{L F}$ as $\bar{\phi}_{L F}\left(x_{\perp}, x^{-}\right)$, and computing the matrix element yields the result

$$
\bar{\phi}_{L F}\left(x_{\perp}, x^{-}\right)=\left\langle\psi_{0}\left|\phi\left(r_{\perp}=x_{\perp}, r_{3}=-x^{-} v^{+}\right)\right| \psi_{0}\right\rangle,
$$

in which the latter matrix element is given in Eq. (2.14). Such a relation should be useful in constructing a more elaborate theory.

\section{MESON DISTRIBUTION IN THE LF FORMULATION - VECTOR MESONS}

The calculation of vector meson distributions is based on the formalism in Ref. [6], which used earlier work [13]. In the present work, we take the vector mesons $V^{\mu}$ to be coupled to a large nuclear source of baryon current $J^{\mu}$. Thus the relevant Lagrangian density $\mathcal{L}_{V}$ is given by

$$
\mathcal{L}_{V}=-\frac{1}{4} V^{\mu \nu} V_{\mu \nu}+\frac{m_{V}^{2}}{2} V^{\mu} V_{\mu}-J^{\mu} \bar{V}_{\mu}
$$

where the bare mass of the vector mesons given by $m_{V}$, and $V^{\mu \nu}=\partial^{\mu} V^{\nu}-\partial^{\nu} V^{\mu}$ and [6]

$$
\bar{V}^{\mu}=V^{\mu}-\partial^{\mu} \frac{1}{\partial^{+}} V^{+}
$$

so that $\bar{V}^{+}=0$.

The essential points which simplify our analysis in the mean field approximation are

- The vector current is proportional to the 4-velocity of the source, so that one finds

$$
\tilde{J}_{L F}^{\mu}\left(\vec{k}_{\perp}, k^{+}\right)=v^{\mu} \tilde{J}_{R F}^{V}\left(\vec{k}_{\perp}, k^{+} / v^{+}\right)
$$

where

$$
J_{R F}^{V}=g_{V} \rho_{B} \Theta(R-r)
$$

which has the same coordinate dependence as the model for $\tilde{J}_{R F}$ used for scalar mesons and $g_{V}$ is the vector meson coupling constant.

- We work in a frame where $\vec{v}_{\perp}=0$, and thus $\vec{J}_{\perp}=0$.

- As explained in Ref. [6], we work with degrees of freedom such that the + component of the vector meson field vanishes.

This allows one to obtain the relevant Hamiltonian, which can then be solved by means of a coherent state ansatz analogously to the case of scalar mesons.

The main difference between vector mesons and scalar mesons is the appearance of the polarization vector $\bar{\varepsilon}^{\mu}$ in the coupling of vector mesons to the nucleon current [6]

$$
\begin{array}{r}
\bar{V}^{\mu}=\int_{0}^{\infty} \frac{d k^{+}}{\sqrt{4 \pi k^{+}}} \int \frac{d^{2} k_{\perp}}{2 \pi} \sum_{\omega=1,3} \bar{\varepsilon}^{\mu}(\vec{k}, \omega) \times \\
{\left[a_{k^{\dagger} \vec{k}_{\perp}, \omega} e^{i \boldsymbol{k} \cdot \boldsymbol{x}}+a_{k^{+} \vec{k}_{\perp}, \omega} e^{-i \boldsymbol{k} \cdot \boldsymbol{x}}\right]}
\end{array}
$$

where $a_{k+\vec{k}_{\perp}, \omega}$ satisfies standard boson commutation relations and $\omega$ labels the polarization states. Since the transverse components of the nucleon current vanishes in our model, and since $\bar{\varepsilon}^{+}=0$, the only relevant component of $\bar{\varepsilon}^{\mu}$ is $\mu=-$.

The coherent state takes the form 


$$
\left|\psi_{0}^{V}\right\rangle \propto\left[\prod_{k^{+}, \vec{k}_{\perp}} \exp \left(\frac{\tilde{J}_{R F}^{\mu}\left(\vec{k}_{\perp}, k^{+} / v^{+}\right)^{*} \bar{\varepsilon}_{\mu} a_{k^{+} \vec{k}_{\perp}}^{\dagger}, \omega}{v^{+} \sqrt{2 k^{+}}\left(\frac{m_{V}^{2}+\vec{k}_{\perp}^{2}}{2 k^{+}}+\frac{k^{+}}{2 v^{+2}}\right)}\right)\right]|0\rangle .
$$

One may use this state to take matrix elements of the plus-momentum operator and the light front Hamiltonian. These expressions are similar to those for the scalar mesons except that a factor of $\left(v^{+} \bar{\varepsilon}^{-}\right)^{2}$ summed over all polarization states is present. The polarization sum is given by [6]

$$
\sum_{\omega=1,3} \bar{\varepsilon}^{-}(\vec{k}, \omega) \bar{\varepsilon}^{-}(\vec{k}, \omega)=\frac{m_{V}^{2}+\vec{k}_{\perp}^{2}}{k^{+^{2}}} .
$$

The light front Hamiltonian consists of three terms: the kinetic energy (Eq. (2.24) of Ref. [6]); the linear coupling and, the effects of the instantaneous vector meson exchange. Thus the light-front energy of the nucleus due to coupling to the vector meson field consists of two pieces. First there is a contribution which arises from physical vector meson intermediate states, obtained from the expectation value of the kinetic energy and linear terms. This piece differs from the meson momentum distribution by one power of the energy denominator. In addition to this piece, the unphysical components of the vector meson field contributes an instantaneous selfinteraction of the nucleon field, which exactly cancels the most infrared singular terms of the term due to dynamical mesons. This instantaneous term is very much analogous to the Coulomb interaction for an electromagnetic field. This term is obtained by canonical light front quantization and e.g. is included in Eq. (2.48) of Ref. [6].

One takes the matrix element of the light front Hamiltonian to obtain the ground state energy of the vector meson field coupled to the fixed source:

$$
\begin{aligned}
P_{0}^{-} & =P_{\text {inst }}^{-}-\frac{1}{v^{+2}} \int_{0}^{\infty} \frac{d k^{+}}{2 k^{+}} \int d^{2} k_{\perp} \frac{\left.\frac{m_{V}^{2}+\vec{k}_{\perp}^{2}}{k^{+2}} \mid \tilde{J}_{L F}^{+}\left(\vec{k}_{\perp}, k^{+}\right)\right)\left.\right|^{2}}{\frac{m_{V}^{2}+\vec{k}_{\perp}^{2}}{2 k^{+}}+\frac{1}{2} \frac{k^{+}}{v^{+2}}} \\
& =P_{\text {inst }}^{-}-\int_{0}^{\infty} \frac{d k^{+}}{2 k^{+}} \int d^{2} k_{\perp} \frac{\left.\frac{m_{V}^{2}+\vec{k}_{\perp}^{2}}{k^{+2}} \mid \tilde{J}_{R F}^{V}\left(\vec{k}_{\perp}, k^{+} / v^{+}\right)\right)\left.\right|^{2}}{\frac{m_{V}^{2}+\vec{k}_{\perp}^{2}}{2 k^{+}}+\frac{1}{2} \frac{k^{+}}{v^{+2}}},
\end{aligned}
$$

where we used Eq. (4.3) and where the instantaneous self-interaction is given by

$$
\left.P_{\text {inst }}^{-}=\int_{0}^{\infty} \frac{d k^{+}}{k^{+^{2}}} \int d^{2} k_{\perp} \mid \tilde{J}_{L F}^{+}\left(\vec{k}_{\perp}, k^{+}\right)\right)\left.\right|^{2} .
$$

Note that the instantaneous piece [Eq. (4.9) can be combined with the rest in Eq. (4.8) yielding

$$
P_{0}^{-}=\frac{1}{v+^{2}} \int_{0}^{\infty} \frac{d k^{+}}{2 k^{+}} \int d^{2} k_{\perp} \frac{\left|\tilde{J}_{R F}^{V}\left(\vec{k}_{\perp}, k^{+} / v^{+}\right)\right|^{2}}{\frac{m_{V}^{2}+k_{\perp}^{2}}{2 k^{+}}+\frac{1}{2} \frac{k^{+}}{v^{+2}}},
$$

which is related to the vector meson contribution to the binding energy $P_{0}^{-} v^{+}=E_{R F}^{V}$. The quantity $E_{R F}^{V}$, is up to a sign (reflecting the fact that scalar meson give rise to attraction, while vector mesons give rise to repulsion between nucleons) of the same form as the scalar result Eq. (3.8).

The result of taking matrix elements of the plusmomentum operator in the coherent state leads to the result:

$$
\begin{aligned}
& \rho_{V}\left(\vec{k}_{\perp}, k^{+}\right)= \frac{1}{2 k^{+}} \frac{\frac{m_{V}^{2}+\vec{k}_{\perp}^{2}}{k^{+2}}\left|\tilde{J}_{R F}^{V}\left(\vec{k}_{\perp}, k^{+} / v^{+}\right)\right|^{2}}{\left[\frac{m_{V}^{2}+\vec{k}_{\perp}^{2}}{2 k^{+}}+\frac{1}{2}\left(\frac{k^{+}}{v^{+2}}\right)^{2}\right]^{2}} \\
&=\left|\tilde{J}_{R F}^{V}\right|^{2}\left\{\frac{2}{k^{+}} \frac{1}{m_{V}^{2}+\vec{k}_{\perp}^{2}+\left(\frac{k^{+}}{v^{+}}\right)^{2}}\right. \\
&\left.\quad-\frac{2 k^{+}}{v^{+2}} \frac{1}{\left[m_{V}^{2}+\vec{k}_{\perp}^{2}+\left(\frac{k^{+}}{v^{+}}\right)^{2}\right]^{2}}\right\} .
\end{aligned}
$$

The second term on the r.h.s. of Eq. (4.11) is (up to a sign and a differing mass) identical to the momentum distribution of scalar mesons. The contribution of this second term to the momentum (per nucleon) carried by the vector mesons vanishes in the nuclear matter limit and explicit numerical evaluation shows that for finite nuclei it is negligible compared with the the first term. This singular term

$$
\rho_{V}^{\text {sing }}\left(\vec{k}_{\perp}, k^{+}\right) \equiv \frac{2\left|\tilde{J}_{R F}^{V}\right|^{2}}{k^{+}} \frac{1}{m_{V}^{2}+\vec{k}_{\perp}^{2}+\left(\frac{k^{+}}{v^{+}}\right)^{2}}
$$

is more interesting since it diverges as $k^{+} \rightarrow 0$. By direct comparison one can verify that the contribution from this term to the total momentum carried by the vector mesons is (up to a factor $v^{+}$) identical to the contribution of the vector mesons to the rest-frame energy of the nucleus

$$
\begin{aligned}
\int_{0}^{\infty} d k^{+} \int d^{2} k_{\perp} \rho_{V}^{\text {sing }} k^{+} & =\int_{0}^{\infty} d k^{+} \int d^{2} k_{\perp} \frac{2\left|\tilde{J}_{R F}\right|^{2}}{m_{V}^{2}+\vec{k}_{\perp}^{2}+\left(\frac{k^{+}}{v^{+}}\right)^{2}} \\
& =v^{+2} P_{0}^{-}=v^{+} E_{R F}^{V}
\end{aligned}
$$

Together with the result from the previous section that the non-singular piece does not contribute to the momentum per nucleon in the nucleus for infinite nuclear matter one thus finds that in the infinite nuclear matter limit the momentum carried by the vector mesons is given by

$$
\left\langle k_{V}^{+}\right\rangle=v^{+} E_{R F},
$$

i.e. the momentum fraction carried by the vector mesons is given by the ratio between the meson interaction energy and the mass of the nucleus 


$$
\left\langle x_{V}\right\rangle=\frac{\langle V\rangle}{M_{A}}
$$

Note that the potential energy $\langle V\rangle$ on the right hand side of Eq. (4.15) is only the part due to vector mesons. This result (momentum carried by vector mesons equals potential energy due to vector mesons) holds regardless whether or not there is also a scalar interaction present. Since both scalar and vector interaction are rather large in nuclei (of opposite sign, such that their net effect is small), this means that vector mesons may carry a substantial fraction of the nucleus' momentum. In the previous section, we have shown that scalar mesons carry only a small fraction so the net momentum carried by the mesons is essentially the momentum carried by the vector mesons, which can be very large.

\section{A. Vector Meson Plus-Momentum Distribution}

The result that vector mesons carry a large part of the nuclear plus-momentum, which had also been predicted in Ref. [6] using more general arguments, is at first surprising since the vector meson field (very much like the scalar meson field) becomes space independent for nuclear matter in the mean field approximation. Therefore, one would expect that the vector meson field for nuclear matter contains only quanta with vanishing + momentum. It thus seems paradoxical that vector mesons nevertheless carry a finite fraction of the nucleus' + momentum in this limit.

In order to resolve this apparent paradox, let us analyse the momentum distribution arising from the crucial singular piece $\rho_{V}^{\text {sing }}$ of Eq. (4.12) more closely.

The dominant, singular part of the vector meson lightfront momentum density depends on the source

$$
\tilde{J}_{R F}^{V}\left(\vec{k}_{\perp}, k^{+} / v^{+}\right)=g_{V} \rho_{B} \sqrt{\frac{2}{\pi}} R^{3} \frac{j_{1}(k R)}{k R}
$$

with

$$
k \equiv \sqrt{\vec{k}_{\perp}^{2}+\frac{k^{+^{2}}}{v^{+^{2}}}} .
$$

We define a + -momentum distribution function per nucleon $\left(A=\frac{4 \pi}{3} R^{3} \rho_{B}\right)$, using the $x$-variable of Eq. (3.16) so that

$$
f_{V}(x) \equiv \frac{3 M_{N} v^{+}}{4 \pi R^{3} \rho_{B}} \int d^{2} k_{\perp} \rho_{V}^{s i n g}\left(\vec{k}_{\perp}, k^{+}\right) .
$$

One may simplify the integral such that

$$
x f_{V}(x)=R^{3} \frac{6}{\pi} g_{V}^{2} \rho_{B} \int_{x M_{N} R}^{\infty} \frac{d y}{y} \frac{1}{y^{2}+m_{V}^{2} R^{2}} j_{1}^{2}(y) .
$$

A qualitative understanding may be gained by noting that the quantity $j_{1}^{2}(y) / y$ peaks at $y \approx 1.6$, so that the denominator can be simplified by treating $\frac{1}{y^{2}+m_{V}^{2} R^{2}} \approx$ $\frac{1}{m_{V}^{2} R^{2}}$ and therefore

$$
x f_{V}(x) \approx \frac{R}{m_{V}^{2}} \frac{6}{\pi} g_{V}^{2} \rho_{B} \int_{x M_{N} R}^{\infty} \frac{d y}{y} j_{1}^{2}(y) .
$$

The integral vanishes in the limit $R \rightarrow \infty$ for all $x \neq 0$. In particular, for $x M_{N} R \gg 1$ an upper limit may be obtained by approximating $j_{1}(y) \approx \cos y / y \geq 1 / y$. Thus the integral is less than $\frac{1}{2}\left(\frac{1}{x M_{N} R}\right)^{2}$, so that for non-zero values of $x$,

$$
x f_{V}(x) \sim \frac{R}{\left(x M_{N} R\right)^{2}},
$$

which vanishes as $R$ approaches $\infty$. If $x=0$, the integral takes on the value $1 / 3$. Thus in the limit of infinite $R$ there is a sharp distinction between the results for $x=$ 0 and for non-zero values, no matter how small. This suggests that $\lim _{R \rightarrow \infty} x f_{V}(x)$ might be a delta function.

To proceed further we need to show that

$$
\lim _{R \rightarrow \infty} \int_{0}^{x_{U}} d x x f_{V}(x)=\left(\frac{g_{V}}{m_{V}}\right)^{2} \frac{\rho_{B}}{M_{N}}
$$

and

$$
\lim _{R \rightarrow \infty} \int_{x_{L}}^{x_{U}} d x x f_{V}(x)=0
$$

for $x_{L}>0$. The above two equations would mean that

$$
\lim _{R \rightarrow \infty} x f_{V}(x)=\left(\frac{g_{V}}{m_{V}}\right)^{2} \frac{\rho_{B}}{M_{N}} \delta(x)
$$

in accord with the result expected from earlier work. The present meaning of the function $\delta(x)$ is that integrals over $x$ including this delta function are non-vanishing provided the lower limit is infinitesimally close to zero.

To verify Eq.(4.22) it is simply necessary to do the integral. One interchanges the order of integration, takes $R \rightarrow \infty$ and uses the upper limit of the integral of Eq. 4.20). Then the result is immediate if one uses the integral

$$
\int_{0}^{\infty} j_{1}^{2}(x) d x=\frac{\pi}{6} .
$$

The verification of Eq.(4.23) also proceeds by integration and by using Eqs. (4.20) and (4.21). Thus Eq. (4.24) is valid and in infinite nuclear matter the quantity $x f_{V}(x)$ has support only at $x=0$, or $k^{+}=0$.

The next step is to study the distribution function $f_{V}(x)$ of Eq.4.19). First, we calculate the momentum fraction carried by the vector mesons

$$
\begin{aligned}
\left\langle x_{V}\right\rangle & \equiv \int d x x f(x) \\
& =\frac{1}{M_{N} \frac{4 \pi}{3} R^{3} \rho_{B}} \int d^{3} k \frac{\left|\tilde{J}_{R F}(\vec{k})\right|^{2}}{m_{V}^{2}+\vec{k}^{2}}
\end{aligned}
$$


The integral is the same one that appears in the calculation of the vector meson contribution ot the binding energy, showing that $\left\langle k^{+}\right\rangle=v^{+} E_{R F}$ for the vector meson contributions. We take the limit of infinite nuclear matter to find

$$
\begin{aligned}
\left\langle x_{V}\right\rangle & \stackrel{R \rightarrow \infty}{\longrightarrow} \frac{1}{M_{N} \frac{4 \pi}{3} R^{3} \rho_{B}} \int d^{3} k \frac{\left|\tilde{J}_{R F}(\vec{k})\right|^{2}}{m_{V}^{2}} \\
& =\frac{g_{V}^{2} M_{N}^{2}}{m_{V}^{2}} \frac{\rho_{B}}{M_{N}^{3}}
\end{aligned}
$$

We follow Ref [6] and use the parameters of of Chin and Walecka [12

$$
\frac{g_{V}^{2} M_{N}^{2}}{m_{V}^{2}}=195.9, \quad \rho_{B}=.193 \mathrm{fm}^{-3} .
$$

Then $\left\langle x_{V}\right\rangle=0.348$, which agrees with the result of Ref [6.

Numerical studies of $x f_{V}(x)$ of Eq. (4.19) for finite values of $R$ are shown in Fig. 2.

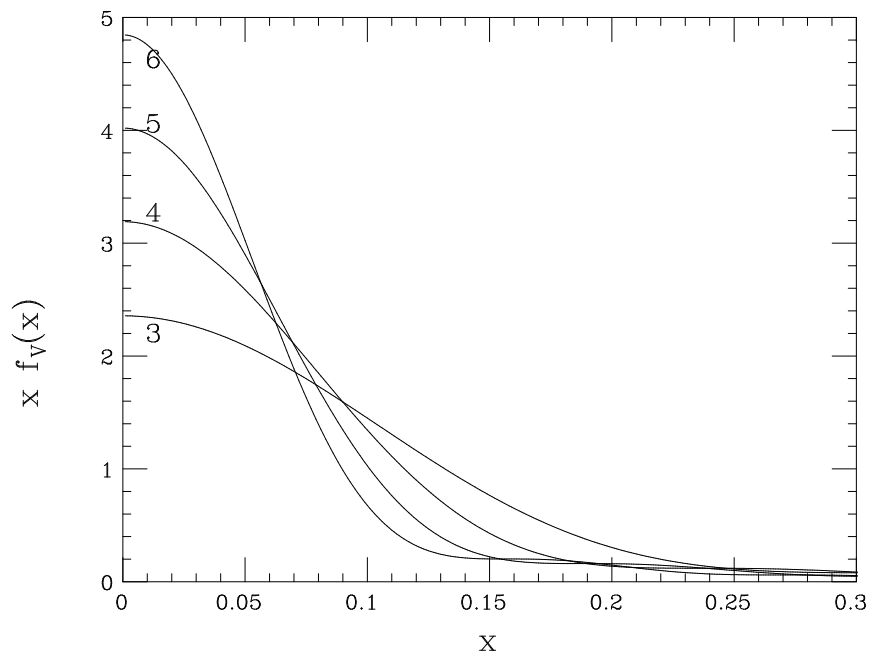

FIG. 2. The quantity $x f_{V}(x)$ vs. $x$ for nuclei of radii $3,4,5,6$ $\mathrm{fm}$ (the numbers on the curves refer to the nuclear radius ).

The results of Fig. 2 2 show the typical behavior of distributions that approach delta functions. The approach to the delta function limit is slow, as expected from the $1 / R$ behavior displayed in Eq. (4.21). Indeed, all of the results show a significant spread and the functions do have some support for values of $x$ such that $x$ greater than about 0.1 . The key physics question we need to address is whether or not the distribution is non-zero for values of $x$ that are too small to be observable. This does happen in the limit of infinite nuclear matter $R \rightarrow \infty$. We may examine this question in another way by considering how much of the total vector meson plus momentum occurs in an observable region. We arbitrarily define the low $x$ (un-observable) region here as $x<0.1$. The distribution functions shown here are to be convoluted with a vector-meson quark-distribution function, which peaks (qualitatively) at $1 / 2$. Thus $x<0.1$ corresponds to $x_{B j}$ less than about 0.05 . That value is small enough so that we can say the vector mesons would be hidden by the effects of shadowing. Even in that case we see qualitatively from Fig. (2) and by computation that approximately $0.05(\mathrm{R}=6 \mathrm{fm})$ and $0.08(\mathrm{R}=3 \mathrm{fm})$ of the total nuclear plus momentum occurs in the observable region of $x_{B j}>0.05$. Thus the mean field vector mesons could be observable in this model. We note that the parameters used here have been shown to give a much larger nucleon depletion than allowed by data. Thus the current results provide an overestimate.

\section{SUMMARY}

We have explicitly constructed the scalar and vector meson states for a mean field model of large nuclei and have obtained some rigorous results for the meson distribution functions.

Direct calculation of the light-front momentum distribution for scalar mesons in the mean field approximation is localized near $k^{+} \rightarrow 0$. As a result, the total momentum carried by the scalar mesons in the mean field approximation is vanishingly small for nuclear matter. This calculation thus confirms the results of Ref. [6]: even though scalar mesons contribute to the nuclear binding in nuclear matter, they carry only a vanishing fraction of the momentum in the mean field calculation.

Also in accord with Ref. [6] is the present result that vector mesons do contribute to the plus-momentum of the nucleus in the same limit of infinite nuclear matter. In fact, we show that the momentum fraction carried by vector mesons in mean field approximation and in the nuclear matter limit is given by the ratio between the the vector meson contribution to the potential energy and the nuclear mass. Surprisingly, this result is obtained despite the fact that the vector meson distribution functions are zero for non-zero $x_{B j}$ for infinitely large nuclei. This is because the vector meson distribution function is sharply peaked such that $x f_{V}(x) \propto \delta(x)$.

For nuclear radii $R$ corresponding to realistic large nuclei, the meson distributions, while strongly peaked at low values of $x$, are wide enough so that some fraction of the mesons cold be observable. Thus the strict zeros, of Ref. [6], are not obtained. However, the predictive power of the momentum sum rule is still vitiated because a significant fraction of the mesons are hidden at small values of $x_{B j}$.

\section{APPENDIX A: DERIVATION OF THE RECOIL LIGHT FRONT HAMILTONIAN}

This Appendix is concerned with the derivation of Eq. (3.5). This is implicit in the work of Ref. [8] and 
also in Ref. 14, but the importance of this term makes it relevant to derive it for the current nuclear context.

Consider a situation in which a nucleus $\left|\Psi_{A}\right\rangle$ consists of one heavy source of mass $M_{H}$ and light degrees of freedom $L$. The light front momentum and energy eigenvalues are $P_{A}^{ \pm}=M_{A} v^{ \pm}$with $v^{+} v^{-}=\frac{1}{2}$. It is worthwhile to remove the large mass $M_{H}$ from the eigenvalue. Thus we follow Ref. [8] and write

$$
M_{A}=M_{H}+\delta E
$$

where $\delta E$ is the binding energy. The light front eigenvalue equation $M_{A} v^{-}\left|\Psi_{A}\right\rangle=\widehat{P}^{-}\left|\Psi_{A}\right\rangle$ then reads

$$
\begin{aligned}
& \frac{M_{H}+\delta E}{2 v^{+}}\left|\Psi_{A}\right\rangle \\
& \quad=\int d^{2} k_{\perp} d k^{+} \frac{k_{\perp}^{2}+M_{H}^{2}}{2 k^{+}} A^{\dagger}\left(k_{\perp}, k^{+}\right) A\left(k_{\perp}, k^{+}\right)\left|\Psi_{A}\right\rangle \\
& \quad+\left(\widehat{P}_{H L}^{-}+\widehat{P}_{L L}^{-}\right)\left|\Psi_{A}\right\rangle
\end{aligned}
$$

where $A\left(k_{\perp}, k^{+}\right)$is the destruction operator of the heavy source, and the light front Hamiltonian for the interaction between the heavy and light degrees of freedom is $\widehat{P}_{H L}^{-}$ and that for the light degrees of freedom is $\widehat{P}_{L L}^{-}$. One would like to remove the large mass $M_{H}$ from both sides of the equation. This may be accomplished by making the replacement

$$
k^{+} \rightarrow P_{A}^{+}-\widehat{P}_{L}^{+}=M_{H} v^{+}\left(1+\frac{\delta E v^{+}-\widehat{P}_{L}^{+}}{M_{H} v^{+}}\right)
$$

in Eq. (A2), where $\widehat{P}_{L}^{+}$is the second-quantized kinematicplus-momentum of the light degrees of freedom. Using the above replacement, and ignoring terms of order $M_{H}^{-1}$ leads to the replacement

$$
\frac{k_{\perp}^{2}+M_{H}^{2}}{k^{+}} \rightarrow \frac{M_{H}}{v^{+}}-\frac{\delta E}{v^{+}}+\frac{\widehat{P}_{L}^{+}}{v^{+^{2}}}
$$

The next step is to remove the heavy source of momentum $k_{\perp}, k^{+}$from $\left|\Psi_{A}\right\rangle$ by acting on it with the operator $A\left(k_{\perp}, k^{+}\right)$. The result is that Eq. (A2) is transformed into

$$
\begin{aligned}
\frac{2 \delta E}{v^{+}} A\left(k_{\perp}, k^{+}\right)\left|\Psi_{A}\right\rangle & (\mathrm{A} 4) \\
= & \left(\frac{\widehat{P}_{L}^{+}}{v^{+^{2}}}+2 \widehat{P}_{H L}^{-}+2 \widehat{P}_{L L}^{-}\right) A\left(k_{\perp}, k^{+}\right)\left|\Psi_{A}\right\rangle .
\end{aligned}
$$

The operator $\frac{\widehat{P}_{L}^{+}}{2 v^{+2}}$ which is the contribution of the recoil momentum of the heavy source to the light front Hamiltonian is the operator that we seek. Note that the quantity in parenthesis and $\frac{2 \delta E}{v^{+}}$are independent of $k_{\perp}, k^{+}$.
[1] J. Aubert et al., Phys. Lett. B 123, 275 (1982); R.G. Arnold, et al.,Phys. Rev. Lett. 52727 (1984); A. Bodek, et al.,Phys. Rev. Lett. 51, 534 (1983).

[2] R.L. Jaffe, in "Relativistic Dynamics and Quark-Nuclear Physics", Ed. by M. B. Johnson and A. Picklesimer (Wiley, New York, 1985); L.L. Frankfurt and M.I. Strikman, Phys. Rep. 160235 (1988); M. Arneodo, Phys. Rep. 240, 301 (1994). D.F. Geesaman, K. Saito, A.W. Thomas, Ann. Rev. Nucl. Part. Sci. 45, 337 (1995).

[3] R.P. Bickerstaff, M.C. Birse, and G.A. Miller, Phys. Rev. Lett.53, 2532 (1984). M. Ericson, and A.W. Thomas, Phys. Lett. 148B, 191 (1984); E.L. Berger, Nucl. Phys. B267 231 (1986).

[4] D.M. Alde et al. Phys. Rev. Lett. 64, 2479 (1990).

[5] G.F. Bertsch, L. Frankfurt, and M. Strikman, Science 259, 773 (1993).

[6] G.A. Miller, Phys. Rev. C56 R8, (1997); G.A. Miller, Phys. Rev. C56 2789, (1997).

[7] B.D. Serot and J.D. Walecka, Adv. Nucl. Phys. 16 (1986); IU-NTC-96-17, Jan. 1997 nucl-th/9701058

[8] M. Burkardt, in "Theory of Hadrons and Light-Front QCD", Ed. S. Glazek, (World Scientific, Singapore, 1994).

[9] M. Burkardt, Advances Nucl. Phys. 23, 1 (1996).

[10] M. Ericson and A.W. Thomas, Phys. Lett. B132, 112 (1983).

[11] H. Jung and G.A. Miller, Phys. Rev. C41, 659 (1990).

[12] S. A. Chin, and J.D. Walecka, Phys. Lett. B52, 24 (1974).

[13] D.E. Soper, Phys. Rev. D4, 1620 (1971); T-M Yan, Phys. Rev. D7, 1760 (1974).

[14] S. Glazek and R.J. Perry, Phys. Rev. D45, 3734 (1992). 